\title{
Energy-Optimal Braking Control Using a Double- Layer Scheme for Trajectory Planning and Tracking of Connected Electric Vehicles
}

Hao-xuan Dong

Southeast University

Wei-chao Zhuang

Anhui Medical University

Guo-dong Yin ( $\nabla$ ygd@seu.edu.cn )

Southeast University https://orcid.org/0000-0002-8437-7598

Li-wei Xu

Southeast University

Yan Wang

Southeast University

Fa-an Wang

Southeast University

Yan-bo Lu

Southeast University

\section{Original Article}

Keywords: Connected electric vehicles, Energy optimization, Velocity planning, Regenerative braking, Dynamic programming, Model predictive control

Posted Date: June 30th, 2020

DOI: https://doi.org/10.21203/rs.3.rs-37338/v1

License: (1) (1) This work is licensed under a Creative Commons Attribution 4.0 International License. Read Full License

Version of Record: A version of this preprint was published at Chinese Journal of Mechanical Engineering on August 31st, 2021. See the published version at https://doi.org/10.1186/s10033-021-00601-3. 


\section{Title page}

\section{Energy-Optimal Braking Control Using a Double-Layer Scheme for Trajectory Planning and Tracking of Connected Electric Vehicles}

Hao-xuan Dong, born in 1993, is currently a PhD candidate at the School of Mechanical Engineering, Southeast University, China. He received his M.S. degree on vehicle engineering from Chang'an University, China, in 2018. His research interests include connected vehicles, vehicle energy management, eco-driving control.

Tel: +86-18363623668; E-mail: donghaox@foxmail.com

Wei-chao Zhuang, born in 1990, is currently an Assistant Professor at the School of Mechanical Engineering, Southeast University, China. He received the B.S. and Ph.D. degrees on mechanical engineering from Nanjing University of Science and Technology, China, in 2012 and 2017, respectively. His current research interests include vehicle dynamics and control, optimal control, clean energy vehicles, connected vehicles, and multi-agent control.

Tel: +86-15951860337; E-mail: wezhuang@seu.edu.cn

Guo-dong Yin, born in 1976, is currently a Professor at the School of Mechanical Engineering, Southeast University, China. He received the Ph.D. degree on vehicle engineering from Southeast University, China, in 2007. His current research interests include vehicle dynamics and control, connected vehicles, and multiagent control.

Tel: +86-13913879060; E-mail: ygd@seu.edu.cn

Li-wei Xu, born in 1986, is currently a Research Associate at the School of Mechanical Engineering, Southeast University, China. He received the Ph.D. degree on mechanical engineering from Southeast University, China, in 2019. His current research interests include vehicle dynamics and control, autonomous vehicles, and connected vehicles.

Tel: +86-18602548629; E-mail: eagil123@hotmail.com

Yan Wang, born in 1992, is currently a PhD candidate at the School of Mechanical Engineering, Southeast University, China. He received the M.S. degree on vehicle engineering from Nanjing University of Aeronautics and Astronautics, in 2018. His current research interests include vehicle state and parameter estimation, automotive active safety control.

Tel: +86-13645165076; E-mail: yanwangiv@outlook.com

Fa-an Wang, born in 1990, is currently a PhD candidate at the School of Mechanical Engineering, Southeast University, China. He received the M.S. degree on agricultural engineering from Kunming University of Science and Technology, China, in 2017. His current research interests include vehicle cooperative localization and control, intelligent connected vehicles.

Tel: +86-18317710956; E-mail: faanwang@seu.edu.cn

Yan-bo Lu, born in 1995, is currently a PhD candidate at the School of Mechanical Engineering, Southeast University, China. He received his B.S. degree on mechanical engineering from Nanjing University of Aeronautics and Astronautics, China, in 2017. His research interests include vehicle dynamics and control, vehicle rollover prevention.

Tel: +86-13451826598; E-mail: 230198035@seu.edu.cn

\section{Corresponding author: Guo-dong Yin E-mail: ygd@seu.edu.cn}




\title{
Energy-Optimal Braking Control Using a Double-Layer Scheme for Trajectory Planning and Tracking of Connected Electric Vehicles
}

\author{
Hao-xuan Dong ${ }^{1} \bullet$ Wei-chao Zhuang ${ }^{1} \bullet$ Guo-dong Yin ${ }^{1} \bullet$ Li-wei Xu ${ }^{1} \bullet$ Yan Wang$^{1} \bullet$ Fa-an Wang ${ }^{1} \cdot{\text { Yan-bo } \text { Lu}^{1}}^{1}$
}

Received June xx, 201x; revised February xx, 201x; accepted March xx, 201x

(C) Chinese Mechanical Engineering Society and Springer-Verlag Berlin Heidelberg 2017

\begin{abstract}
'Abstract: To improve the energy efficiency of electric vehicles (EVs), this paper presents an energy-optimal braking strategy (EOBS) considering shared braking intention. First, a doublelayer control scheme is formulated. In the upper-layer, an energy-optimal braking problem with braking intention is formulated and solved by distance-based dynamic programming, which could derive the energy-optimal braking trajectory. In the lower-layer, a linear time-varying model predictive (LTV-MPC) controller is designed to track the desired trajectory while ensuring braking comfort and safety. Second, several simulations are conducted to demonstrate the regeneration energy efficiency improvement of proposed EOBS. Finally, the energy-optimal braking mechanism of EVs is investigated based on the analysis of braking deceleration, battery charging power, and motor efficiency.
\end{abstract}

Keywords: Connected electric vehicles • Energy optimization • Velocity planning - Regenerative braking - Dynamic programming $\bullet$ Model predictive control

\section{Introduction}

Increasingly stringent fuel economy and emission standards in recent years promote the development of electric vehicles (EVs) [1]. However, the driving range of EVs is still too short compared with the internal combustion engine vehicle, because the battery energy density is insufficient, it is the main obstacles for wide diffusion of EVs [2]. To extend the driving range, one of the popular ways is increasing the battery capacity, however, the battery size and vehicle price are increased simultaneously [3]. Recycling vehicle kinetic energy in the braking process is another prominent approach to extend the driving range, which is called the regenerative braking in some literature [4].

The regenerative braking is the prior choice in some traffic conditions for EVs, e.g., approaching an

Guo-dong Yin ygd@seu.edu.cn intersection with red signal, following the slowed preceding vehicle, and reaching a destination, because the EVs can transfer vehicle kinetic energy to electric energy by regenerative braking while without additional battery energy consumption. Since approximately $30 \% \sim 50 \%$ of driving energy can be recycled in urban traffic [5], several studies have investigated the regenerative braking in recent decades. Some studies tried to optimize the powertrain configuration and vehicle components for increasing regeneration energy. Hellgren et al. [6] demonstrated that the distributed-driven configuration of EVs has superior energy recycle potential than a central driven. Joy et al. [7] presented an integrated power circuit and electronic commutator control strategy to improve the generating energy of EVs. Kiddee et al. [8] designed a hybrid energy storage system, which uses the supercapacitor and lithium-ion battery to enhance the harvesting of braking energy. Zhang et al. [9] proposed an electrical-hydraulic integrated brake configuration based on the electronic stability program to achieve an efficient and safe braking system.

Another approach to improve the regeneration energy is the regenerative braking strategy (RBS) design. The conventional RBS focus on each wheels (axles) braking force distribution, which cooperating the motor braking and friction braking force to improve regeneration energy, while ensuring braking safety, stability, and comfort [10]. Sun et al. [11] integrated the braking control strategy to met with the requirement of high braking stability and energy regeneration rate, which including three braking force operating modes. Xu et.al [12] presented two braking regenerative energy optimization controllers to promote regenerative energy, it considering motor efficiency to distribute the friction and motor braking torque of the front and rear wheels. Li et al. [13] proposed a composite RBS to optimize regenerative and plugging

School of Mechanical Engineering, Southeast University, Nanjing 211189, China 
braking simultaneously with the driver's intention recognition. Lian et al. [14] designed an optimal braking force distribution strategy, while uses a safety distance model to avoid collision. In addition, the rule-based algorithm [9], PID algorithm [15], sliding mode control [16], fuzzy logic control [17], design of experiment method [11], model predictive control (MPC) [12], and dynamic programming (DP) [18] were adopted to refine the RBS.

Recently, the emerging of connected vehicle technologies, i.e., vehicle-to-everything (V2X) communication, provides possibilities to further improve energy efficiency and reduce emission [19]. The traffic, road, and vehicle information can be accessed by V2X, which used to plan the energy-optimal velocity, i.e., the surrounding vehicle states [20], road slope [21], road curvature [22], and traffic signal phase and timing [23]. However, most researches focus on the RBS design in vehicle components control and braking torque distribution, few combine the connected vehicle technologies into braking velocity planning. If the braking intention is accessed by the V2X (i.e., braking distance and terminal velocity), the EVs could plan the braking velocity for recovering more energy.

Therefore, this paper proposes an energy-optimal braking strategy (EOBS), where the key is using shared braking intention to planning energy-optimal braking velocity. The major contributions of this paper are threefold. First, a double layer control scheme is proposed: In the upper-layer, the energy-optimal braking trajectory is planned by the distance-based DP with only one state variable and one control variable for fast computation. Second, to follow the optimal trajectory, the lower-layer designs a tracking controller using the linear time-varying MPC (LTV-MPC), to minimize the trajectory tracking errors. Third, the mechanism of energy-optimal braking is derived. The energy-optimal braking trajectory is consists of multiple phases, which can be converted to a rule-based braking strategy.

The remainder of this paper is organized as follows. Section 2 introduces the EVs model and regenerative braking strategy. Section 3 formulates the energy-optimal braking problem and double-layer EOBS. The EOBS performance is verified and energy-optimal braking mechanism is analyzed in Section 4. Section 5 concludes this paper and proposes future work.

\section{Vehicle model and regenerative braking strategy}

\subsection{Vehicle model}

This paper optimizes the braking velocity of fourwheel-independent-driven electric vehicles, which is powered by four in-wheel motors (IWM).

\subsubsection{Vehicle dynamics}

For energy-optimal braking research, only the longitudinal dynamics is modeled, as shown in Eq. (1). $\dot{x}=f(x, u)=$

$$
\left[\begin{array}{cc}
0 & 1 \\
0 & -\frac{0.5 C_{\mathrm{D}} A_{r} \rho v^{2}+m g f \cos \theta+m g \sin \theta}{\delta m v}
\end{array}\right] x+\left[\begin{array}{c}
0 \\
\frac{1}{\delta m}
\end{array}\right] u
$$

where $m$ is the vehicle mass, $\delta$ is the vehicle rotational inertia coefficient, $g$ is the acceleration of gravity, $\theta$ is the road slope, $f$ is the rolling resistance coefficient, $C_{\mathrm{D}}$ is the aerodynamic drag coefficient, $A_{r}$ is the frontal area, and $\rho$ is the air density. $x=[d v]^{\mathrm{T}}$ is the states variable, $d$ is the distance, $v$ is the velocity. $u=F$ is the control variable, $F$ is the vehicle force, positive for traction force and negative for braking force.

In the traction process, the vehicle force is supplied by IWM, i.e., $F=\sum F_{m}$. In the braking process, the vehicle force including motor force $F_{m}$ and friction braking force $F_{f}$, i.e., $F=\sum F_{m}+\sum F_{f}$. The friction braking force is generated by a hydraulic brake system (HBS). We use the first-order lag relation to represent the HBS dynamic response, i.e.,

$$
\dot{p}_{w}+p_{w}=p_{d}
$$

with

$$
p_{d}=F_{f} r_{w} \kappa
$$

where $p_{\mathrm{w}}$ is the brake wheel cylinder pressure, $\kappa$ is the conversion coefficient of wheel cylinder pressure, $\tau$ is the HBS time constant coefficient, $r_{w}$ is the radius of wheel.

\subsubsection{Energy consumption}

The power of IWM is calculated by Eq. (3).

$$
P_{m}=\frac{F_{m} r_{w} n}{9.55}
$$

where $P_{m}$ is the motor power, positive for propulsion power and negative for generating power. $n$ is the motor rotational speed.

This paper ignores the energy losses in electric wires and considers the auxiliary power $P_{a}$. Then the battery power $P_{b}$ is

$$
P_{b}=\sum P_{m} \eta_{m}^{-\operatorname{sign}\left(P_{m}\right)} \eta_{b}^{-\operatorname{sign}\left(P_{m}\right)}+P_{a} \eta_{b}^{-1}
$$

where $\eta_{b}$ is the battery efficiency, $\eta_{m}$ is the motor efficiency which is determined by the motor torque and speed as shown in Figure 1, sign() is the signum function.

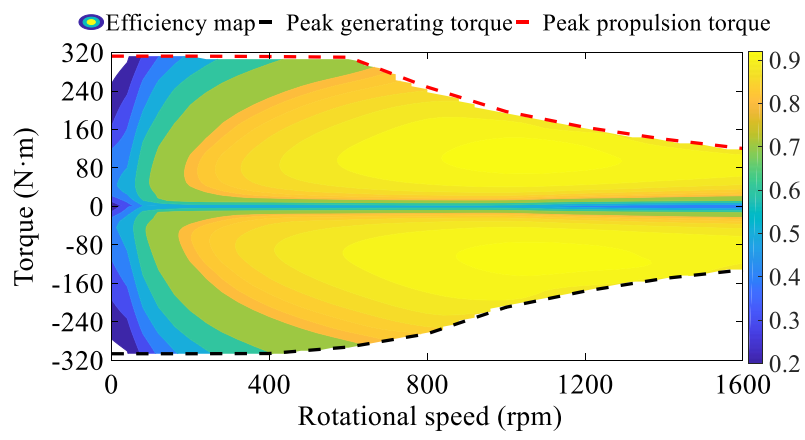

Figure 1 The efficiency map and peak torque of IWM 
The battery is modeled as a simple equivalent circuit [24], which generating current $I_{b}$ is expressed as Eq. (5).

$$
I_{b}=\frac{E-\sqrt{E^{2}-4 R_{o} P_{b}}}{2 R_{o}}
$$

where $E$ is the open-circuit voltage, and $R_{o}$ is the internal resistance. The battery state of charge $(S O C)$ can be calculated by Eq. (6).

$$
S O C=S O C_{0}+\frac{\int I_{\mathrm{b}} d t}{3600 C_{b}}
$$

where $S O C_{0}$ is the battery initial $S O C$ valve, $C_{\mathrm{b}}$ is the battery capacity.

\subsection{Regenerative braking strategy}

The RBS including twofold: braking force distribution on the front and rear axles, motor and friction braking force distribution on each wheels. The distributed braking force on the front and rear axles affect braking stability, then we use 'ideal distribution strategy' to prevent wheel locking [25]:

$$
\begin{gathered}
F_{b f}=\beta F \\
F_{b r}=(1-\beta) F
\end{gathered}
$$

where $F_{b f}$ and $F_{b r}$ are the braking force on the front and rear axle, respectively. $\beta$ is the ideal distribution ratio, which is proportional to vehicle axle normal forces:

$$
\beta=\frac{\left(L_{b}+z h_{g}\right)}{L_{w}}
$$

where $L_{b}$ is the distance from the rear axle to the center of vehicle mass, $L_{w}$ is the vehicle wheelbase, $h_{g}$ is the distance from the ground to the center of vehicle mass, $z$ is the brake strength.

We assume the vehicle driving in the straight road, then the braking force on the left and right wheels are identical. The motor force is preferred to recycle more energy, and HBS provides the remained force while the motor force can not meet the demand. For an example of the front-rear wheel, the motor and friction braking force are calculated by Eqs. (10) and (11).

$$
\begin{gathered}
F_{m}=\min \left(F_{m \max }, 0.5 F_{b f}\right) \\
F_{f}=0.5 F_{b f}-F_{m}
\end{gathered}
$$

where $F_{\operatorname{mmax}}$ is motor maximum braking force.

\section{$3 \quad$ Energy-optimal braking strategy}

This section formulates the energy-optimal braking problem and proposes a double-layer EOBS to improve regeneration energy while ensuring braking comfort and safety. In the upper-layer, the energy-optimal braking trajectory is planned by solving the optimal control problem. In the lower-layer, a tracking controller is used to track the optimized trajectory accurately while ensuring braking comfort and safety. Figure 2 shows the scheme of the double-layer EOBS.

\subsection{Energy-optimal braking trajectory planning}

The energy-optimal braking trajectory is planned by the DP algorithm. Since the DP has been widely used in the energy optimization problem [19], which breaks the optimal control problem into simpler sub-problems and calculates recursively based on the Bellman Optimality Principle [26].

\subsubsection{Optimization problem formulation}

Figure 3 shows the scenario of braking, where $D$ is the distance to the destination (can be a traffic light or a preceding vehicle), $V_{s}$ and $V_{e}$ are the initial and desired terminal velocity. Note that the $D$ and $V_{e}$ are accessed by V2X communication, the $V_{s}$ is obtained using on-board sensors.

In this paper, the braking distance $D$ is fixed, thus the whole problem is discretized in distance-domain by distance step $\Delta d$. The total number of distance $N_{\mathrm{D}}$ is determined by the $D$ and $\Delta d$, i.e., $N_{D}=D / \Delta d-1$. The velocity is selected as the state variable, i.e., $x_{D}=v$, and the braking deceleration $a$ is selected as the control variable, i.e., $u_{D}=a$. Since the distance step is small, the braking deceleration of each sub-problem is assumed to be constant. Thus, the energy-optimal braking problem is expressed in (12) indexed by $k$.

$$
\begin{aligned}
\underset{u_{D} \in U}{\operatorname{Minimum}} & J_{D}\left(x_{D}, u_{D}\right) \\
= & \sum_{k=1}^{k=N_{D}} P_{b}(k) \Delta t_{D}(k)+\alpha\left(v\left(N_{D}\right)-V_{b}\right)^{2}
\end{aligned}
$$

s.t.

$$
\begin{gathered}
x_{D}(0)=V_{s}, \quad x_{D}\left(N_{D}\right)=V_{b} \\
v(k) \in\left[v_{\min }, v_{\max }\right] \\
u_{D}(k) \in\left[a_{\min }, a_{\max }\right]
\end{gathered}
$$

where $v_{\min }$ and $v_{\max }$ are the minimum velocity for efficient passing and the maximum velocity for road limit, respectively. $a_{\min }$ and $a_{\max }$ are the deceleration depend on comfort and vehicle resistance, respectively. $\Delta t_{D}$ is the time interval. Note that the terminal condition of the velocity has been relaxed by converting them to soft constraints and merged into the objective function as Eq. (12), with weighting factor $\alpha$ for the latter term.

Besides, the state transfer of vehicle velocity and time interval in $k$ step are calculated by Eqs. (13) and (14), respectively.

$$
\begin{gathered}
v(k+1)= \begin{cases}\sqrt{v^{2}(k)+2 a(k) \Delta d} & v^{2}(k)+2 a(k) \Delta d>0 \\
0 & v^{2}(k)+2 a(k) \Delta d \leq 0\end{cases} \\
\Delta t_{D}(k)=\frac{v(k+1)-v(k)}{a(k)}
\end{gathered}
$$




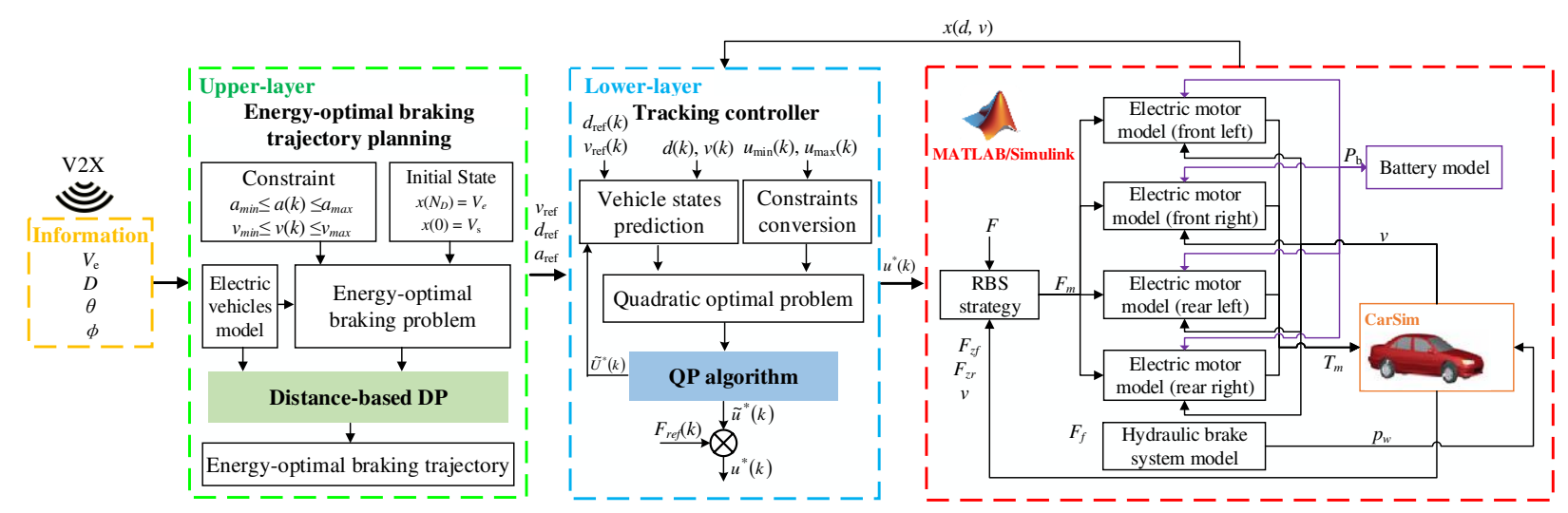

Figure 2 The EOBS scheme

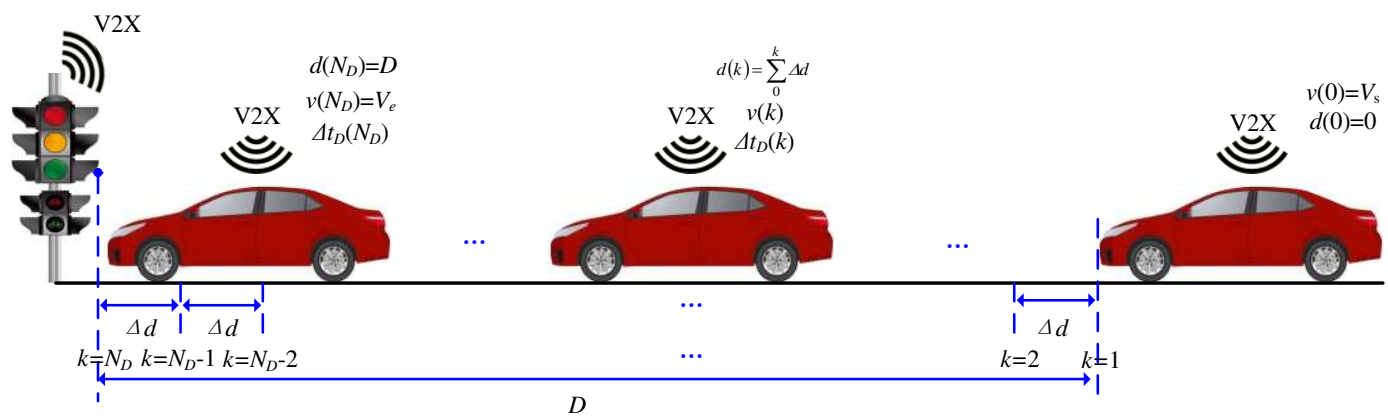

Figure 3 The process of braking

\subsubsection{DP solving}

Denote the transitional cost-to-go function from $k$ step to $k+1$ step $W\left(u_{\mathrm{D}}(k), x_{D}(k)\right)$ and the penalty function for the terminal states $G\left(x_{D}\left(N_{D}\right)\right)$, i.e.,

$$
\begin{gathered}
W\left(u_{D}(k), x_{D}(k)\right)=P_{b}(k) \Delta t_{D}(k) \\
G\left(x_{D}\left(N_{D}\right)\right)=\alpha\left(v\left(N_{D}\right)-V_{s}\right)^{2}
\end{gathered}
$$

The formulated control problem is solved recursively by following the Bellman Optimality Principle [26].

$N_{D}-1$ step:

$$
\begin{aligned}
& J_{N_{D}-1}^{*}\left(x_{D}\left(N_{D}-1\right)\right) \\
& \quad=\min _{u_{D} \in U}\left\{W\left(u_{D}\left(N_{D}-1\right), x_{D}\left(N_{D}-1\right)\right)+G\left(x_{D}\left(N_{D}\right)\right)\right\}
\end{aligned}
$$

$k$ step:

$$
J_{k}^{*}\left(x_{D}(k)\right)=\min _{u_{D} \in U}\left\{W\left(u_{D}(k), x_{D}(k)\right)+J_{k+1}^{*}\left(x_{D}(k+1)\right)\right\}
$$

where $J_{k}{ }^{*}\left(x_{D}(k)\right)$ is the minimum cost-to-go value at the moment $k$. Then, the energy-optimal braking trajectory which starting from any given arbitrary initial and terminal states can be obtained by backward calculation.

\subsection{Tracking controller}

\subsubsection{Tracking problem formulation}

The objective of the tracking controller is to minimize the tracking error between the actual and desired trajectory for ensuring high regeneration efficiency, while adapts to traffic conditions for braking comfort and safety. In this paper, the state error and driving force increment are defined as soft constraints to ensure tracking accuracy and driving comfort, thus, the tracking problem is shown in Eq. (19).

$$
\begin{aligned}
& \underset{u_{M} \in U}{\operatorname{Minimum}} J_{M}\left(u_{M} \mid k\right) \\
& =\sum_{i=1}^{N_{\mathrm{p}}}\|\tilde{x}(k+i \mid k)\|_{Q}^{2}+\sum_{j=1}^{N_{\mathrm{c}}-1}\|\Delta u(k+j \mid k)\|_{R}^{2}+\beta \varepsilon^{2}
\end{aligned}
$$

s.t.

$$
\begin{gathered}
u_{M}(k) \geq u_{\min }(k) \\
D-L \leq d\left(N_{M}\right) \leq D \\
V_{e}-\vartheta \leq v\left(N_{M}\right) \leq V_{e}
\end{gathered}
$$

where $\tilde{x}=\left[d-d_{r e f}, v-v_{r e f}\right]$ is the states tracking error, $d_{\text {ref }}$ and $v_{\text {ref }}$ are the desired distance and velocity, respectively, which are derived from the upper-layer optimization. $\Delta u$ is the increment of the control variable, $\left(u_{M} \mid k\right)$ represents the derived control variable of the controller at $k$ moment. $Q, R$, and $\beta$ are the weighing factor matrix, $\varepsilon$ is the relaxation factor, $N_{p}$ and $N_{c}$ are the prediction horizon and control horizon, respectively. $N_{M}$ is the tracking problem horizon, which determined by the length of the desired trajectory. $L$ is the vehicle length, $\vartheta$ is the velocity slack.

In tracking problem Eq. (19), the constraints including twofold. First, the vehicle force is subject to physical limits $u_{\text {min }}$, which determined by the maximum comfort braking force and road adhesion. Secondly, the virtual stop region that including distance and velocity is defined as a buffer space that relies on safety distance and minimum steady velocity of EVs, then the actual terminal 
Energy-Optimal Braking Control Using a Double-Layer Scheme for Trajectory Planning and Tracking of Connected Electric Vehicles

braking distance and velocity are meet the second and third constraints.

\subsubsection{Linear time-varying state prediction model}

Since the vehicle dynamics in Eq. (1) is nonlinear, it greatly increases the computational burden of MPC. Thus, we employ the approximate linearization method [27] to transform the nonlinear system to a linear system. Thus, the linear time-varying (LTV) vehicle state error model is

$$
\dot{\tilde{x}}=\left[\begin{array}{cc}
0 & 1 \\
0 & -\frac{C_{D} A_{r} \rho v_{r e f}}{\delta m}
\end{array}\right] \tilde{x}+\left[\begin{array}{c}
0 \\
\frac{1}{\delta m}
\end{array}\right] \tilde{u}
$$

where $\tilde{x}=\left[\begin{array}{ll}d-d_{\text {ref }} & v-v_{r e f}\end{array}\right]^{T}, \quad \tilde{u}=\left[F-F_{r e f}\right\rfloor, F_{r e f}$ is the desired braking force. In addition, the MPC calculates the control rule discretely, then the LTV system in Eq. (20) is converted to the discrete-time system using Euler method [28]:

$$
\tilde{x}(k+1)=\left[\begin{array}{cc}
1 & \Delta t_{M} \\
0 & 1-\frac{C_{\mathrm{D}} A_{r} \rho v_{r e f}(k) \Delta t_{M}}{\delta m}
\end{array}\right] \tilde{x}(k)+\left[\begin{array}{c}
0 \\
\frac{\Delta t_{M}}{\delta m}
\end{array}\right] \tilde{u}(k)
$$

where $\Delta t_{M}$ is the time step.

Denote a state prediction matrix:

$$
\varsigma(i+1 \mid k)=\left[\begin{array}{c}
\tilde{x}(i \mid k) \\
\tilde{u}(i-1 \mid k)
\end{array}\right]
$$

where $(i \mid k)$ represents the $i$-step-ahead predicted error at the $k$-th step. Substituting Eq. (21) into Eq. (22), a new state-space model is:

$$
\left\{\begin{array}{c}
\varsigma(i+1 \mid k)=A_{k, i} \varsigma(i \mid k)+B_{k, i} \Delta \tilde{u}(i \mid k) \\
v(i \mid k)=C_{k, i} \varsigma(i \mid k)
\end{array}\right.
$$

with

$$
\begin{aligned}
A_{k, i} & =\left[\begin{array}{ccc}
1 & \Delta t_{M} & 0 \\
0 & 1-\frac{C_{D} A_{r} \rho v_{r e f}(i \mid k) \Delta t_{M}}{\delta m} & \frac{\Delta t_{M}}{m} \\
0 & 0 & 1
\end{array}\right] \\
B_{k, i} & =\left[\begin{array}{lll}
0 & \frac{\Delta t_{M}}{\delta m} & 1
\end{array}\right]^{T} \quad C_{k, i}=\left[\begin{array}{lll}
1 & 0 & 0 \\
0 & 1 & 0
\end{array}\right]
\end{aligned}
$$

Finally, the state prediction model is listed in Eq. (24) by converting Eq. (23).

$$
\tilde{Y}(k)=\Gamma_{k} \varsigma(k)+\Xi_{k} \Delta \tilde{U}(k)
$$

with

$$
\begin{aligned}
& \Gamma_{k}=\left[\begin{array}{lllll}
C_{k, i} A_{k, i} & \cdots & C_{k, i} A_{k, i}^{N_{c}} & \cdots & C_{k, i} A_{k, i}^{N_{p}}
\end{array}\right]_{\mid \times N_{p}} \\
& \Xi_{k}=\left[\begin{array}{cccc}
C_{k, i} B_{k, i} & 0 & \cdots & 0 \\
C_{k, i} A_{k, i} B_{k, i} & C_{k, i} B_{k, i} & \cdots & 0 \\
\vdots & \vdots & \ddots & \vdots \\
C_{k, i} A_{k, i}^{N_{\mathrm{c}}} B_{k, i} & C_{k, i} A_{k, i}^{N_{\mathrm{c}}-1} B_{k, i} & \cdots & C_{k, i} A_{k, i} B_{k, i} \\
\vdots & \vdots & \ddots & \vdots \\
C_{k, i} A_{k, i}^{N_{\mathrm{p}}-1} B_{k, i} & C_{k, i} A_{k, i}^{N_{\mathrm{p}}-2} B_{k, i} & \cdots & C_{k, i} A_{k, i}^{N_{\mathrm{p}}-N_{\mathrm{c}}-1} B_{k, i}
\end{array}\right]_{N_{\mathrm{p}} \times N_{\mathrm{c}}} \\
& \tilde{Y}(k)=\left[\begin{array}{lllll}
v(k+1 \mid k) & \cdots & v\left(k+N_{c} \mid k\right) & \cdots & v\left(k+N_{p} \mid k\right)
\end{array}\right]_{1 \times N_{\mathrm{p}}}^{\mathrm{T}} \\
& \Delta \tilde{U}(k)=\left[\begin{array}{llll}
\Delta \tilde{u}(k \mid k) & \Delta \tilde{u}(k+1 \mid k) & \cdots & \Delta \tilde{u}\left(k+N_{c} \mid k\right)
\end{array}\right]_{1 \times N_{\mathrm{c}}}^{\mathrm{T}}
\end{aligned}
$$

where $\tilde{Y}(k)$ and $\Delta \tilde{U}(k)$ are output and control increment of the prediction model, respectively. Noted that according to the principle of MPC, the $N_{\mathrm{c}} \leq N_{\mathrm{p}}$, and the control input that beyond $N_{\mathrm{c}}$ is always the last control input of $N_{\mathrm{c}}$.

\subsubsection{Quadratic programming solving}

The tracking problem is solved using the quadratic programming (QP) algorithm. Then, the objective function and constraints in Eq. (19) are converted to standard QP form [29], i.e.,.

$$
\begin{aligned}
& J_{M}\left(u_{M} \mid k\right)= \\
& \quad \frac{1}{2}\left[\begin{array}{c}
\Delta \tilde{U}(k)^{\mathrm{T}} \\
\varepsilon
\end{array}\right]\left[\begin{array}{cc}
2\left(\Xi_{k}^{\mathrm{T}} Q \Xi_{k}+R\right) & 0 \\
0 & 2 \beta
\end{array}\right]\left[\begin{array}{c}
\Delta \tilde{U}(k)^{\mathrm{T}} \\
\varepsilon
\end{array}\right]^{\mathrm{T}} \\
& \quad+\left[\begin{array}{c}
2 E_{k}^{\mathrm{T}} Q \Xi_{k} \\
0
\end{array}\right]^{\mathrm{T}}\left[\begin{array}{c}
\Delta \tilde{U}(k)^{\mathrm{T}} \\
\varepsilon
\end{array}\right]^{\mathrm{T}}
\end{aligned}
$$

s.t.

$$
\begin{gathered}
D-L \leq d\left(N_{M}\right) \leq D \\
V_{e}-\vartheta \leq v\left(N_{M}\right) \leq V_{e} \\
{\left[\begin{array}{ccc}
1 & \cdots & 0 \\
\vdots & \ddots & \vdots \\
1 & \cdots & 1
\end{array}\right]_{N_{\mathrm{c}} \times N_{\mathrm{c}}} \Delta \tilde{U}(k)+1_{N \mathrm{c}} \Delta \tilde{u}(k-1) \geq U_{\text {min }}}
\end{gathered}
$$

where $1_{N \mathrm{c}}$ is the column vector with $N_{\mathrm{c}}$ rows, $U_{\min }$ is the minimum value set of control input:

$$
U_{\text {min }}=\left[\begin{array}{llll}
u_{\min }(k) & u_{\min }(k+1) & \cdots & u_{\min }\left(k+N_{\mathrm{c}}-1\right)
\end{array}\right]_{1 \times N_{c}}^{\mathrm{T}}
$$

A series of control input increments $\Delta \widetilde{U}_{K}^{*}$ in the control horizon can be obtained by solving the QP problem. The predicted control input $\tilde{u}^{*}(k)$ is the first term of control sequence plus previous control input $\tilde{u}(k-1)$ :

$$
\tilde{u}^{*}(k)=\tilde{u}(k-1)+\left[\begin{array}{llll}
1 & 0 & \cdots & 0
\end{array}\right]_{1 \times N_{\mathrm{c}}} \Delta \tilde{U}_{k}^{*}
$$

Finally, the predicted vehicle braking force $u^{*}(k)$ is

$$
u^{*}(k)=\tilde{u}^{*}(k)+F_{\text {ref }}(k)
$$

\section{Simulation results and discussion}

In this section, two braking scenarios are simulated to evaluate the regeneration energy efficiency of the proposed strategy, and the constant deceleration braking strategy (CDBS) is simulated as the benchmark. For CDBS [30], the vehicle will brake by fixed deceleration, and the lower-layer uses the same LTV-MPC controller as the proposed strategy to track the fixed deceleration. In addition, the index $\alpha_{r}$ is defined to evaluate the performance of regeneration efficiency as described in Eq. (29) [9]:

$$
\alpha_{r}=\frac{\int P_{b} d t}{0.5 m \delta\left(V_{s}^{2}-v_{e}^{2}\right)} \times 100 \%
$$

where $v_{e}$ is the actual terminal velocity.

\subsection{Simulation setup}

A simulation is conducted by jointing MATLAB 
Energy-Optimal Braking Control Using a Double-Layer Scheme for Trajectory Planning and Tracking of Connected Electric Vehicles

(version 9.4, R2018a) and CarSim (version 20959, 2016.1) on a PC with an Intel Core i7-8700@3.20GHz CPU, the joint simulation structure as shown in Figure 2. The EVs model is established using C-Class hatchback 2012 vehicle in CarSim, and the main parameters of EVs are $f$ $=0.016, m=1421 \mathrm{~kg}, C_{D}=0.3, A_{r}=2.22 \mathrm{~m}^{2}, \rho=1.206$, $r_{w}=0.325 \mathrm{~m}, g=9.8 \mathrm{~m} \cdot \mathrm{s}^{-2}, \delta=1.022, L=4.85 \mathrm{~m}, L_{w}=$ $2.91 \mathrm{~m}, L_{b}=1.9 \mathrm{~m}, h_{g}=0.54 \mathrm{~m}$, and $P_{a}=300 \mathrm{~W}$. The specifications of IWM are the peak propulsion power and generating power are $20.75 \mathrm{~kW}$ and $-20.53 \mathrm{~kW}$, respectively. The peak propulsion torque and generating torque are $312.50 \mathrm{Nm}$ and $-311.50 \mathrm{Nm}$, respectively, and the peak rotational speed is $1600 \mathrm{rpm}$. The specifications of battery are $E=360 \mathrm{~V}, C_{b}=140 \mathrm{Ah}, R_{o}=0.45 \Omega, S O C_{0}$ $=0.8$, and $\eta_{b}=0.9$. According to the HBS module in CarSim, the $\kappa$ of wheels in front axle and rear axle are $250 \mathrm{MPa} /(\mathrm{N} \cdot \mathrm{m})$ and $150 \mathrm{MPa} /(\mathrm{N} \cdot \mathrm{m})$, the $\tau$ of all wheels is 0.06 .

In the DP optimization, the distance interval $\Delta d=0.01 \mathrm{~m}$, the grid step of state variable $v$ and control variable $a$ are $0.1 \mathrm{~m} \cdot \mathrm{s}^{-1}$ and $0.05 \mathrm{~m} \cdot \mathrm{s}^{-1}$, respectively. In LTV-MPC controller, the sampling time is $\Delta t=0.01$, the prediction and control horizon are $N_{p}=100$ and $N_{c}=100$, respectively. The weight matrices are $Q=1000 I_{2 N \mathrm{p} \times 2 N \mathrm{p}}$ and $R=200 I_{N \mathrm{c}}$.

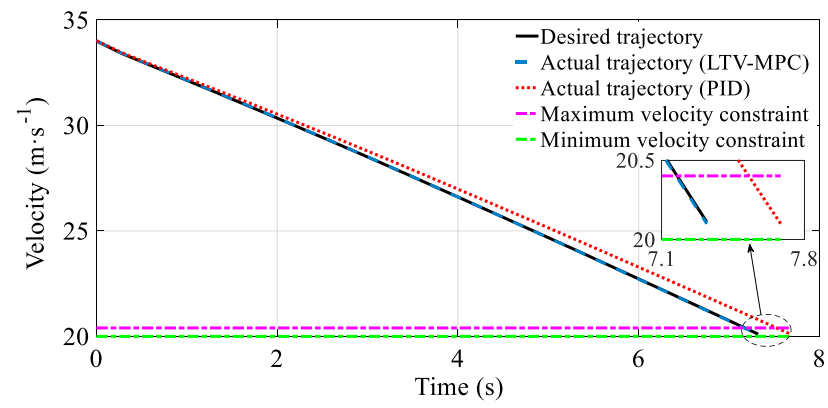

(a) Velocity trajectory
Two scenarios are simulated and referred as Scenario A and Scenario B, i.e., normal braking with small deceleration and emergency braking with big deceleration. The main parameters of these two scenarios are listed in Table 1.

Table 1 Braking scenarios

\begin{tabular}{ccc}
\hline & Scenario A & Scenario B \\
\hline Initial velocity $V_{s}$ & $34 \mathrm{~m} \cdot \mathrm{s}^{-1}$ & $20 \mathrm{~m} \cdot \mathrm{s}^{-1}$ \\
Terminal velocity $V_{e}$ & $20 \mathrm{~m} \cdot \mathrm{s}^{-1}$ & $0 \mathrm{~m} \cdot \mathrm{s}^{-1}$ \\
Distance to destination $D$ & $204 \mathrm{~m}$ & $50 \mathrm{~m}$ \\
Adhesion coefficient $\varphi$ & & 0.85 \\
Velocity slack $\omega$ & & $0.2 \mathrm{~m} \cdot \mathrm{s}^{-1}$ \\
Safety distance slack $\vartheta$ & $4.85 \mathrm{~m}$ \\
Road adhesion $\theta$ & 0 & \\
\hline
\end{tabular}

\subsection{Performance of proposed EOBS}

\subsubsection{Tracking performance}

The tracking performance of the LTV-MPC controller will be verified in Scenario A, and compared with the regular PID controller. Figures 4-6 and Table 2 are the simulation results.

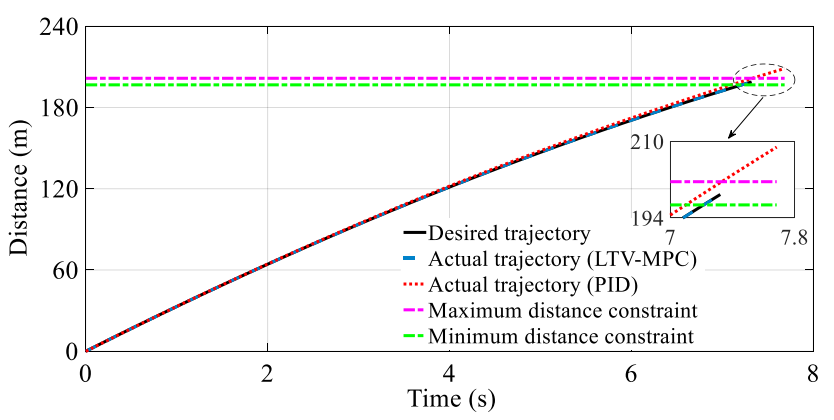

(b) Distance trajectory

Figure 4 The braking trajectory of EVs

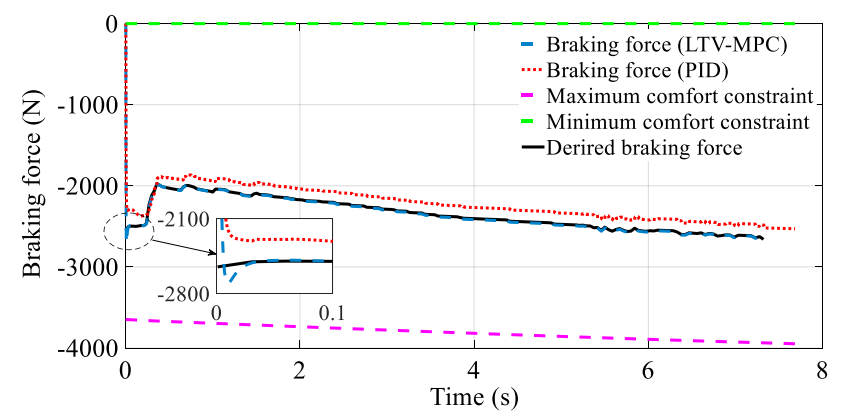

Figure 5 The braking force of EVs

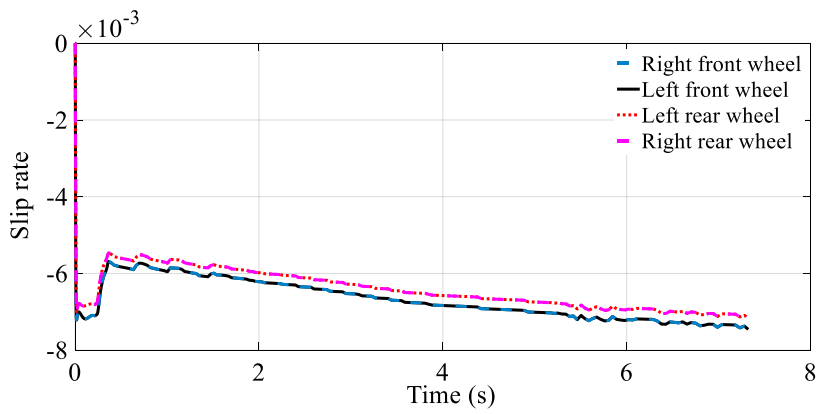

Figure 6 The slip rate of EVs by LTV-MPC

Table 2 Simulation result of LTV-MPC and PID controller

\begin{tabular}{ccccc}
\hline & Regeneration energy efficiency $\alpha_{\mathrm{r}}$ & Braking duration & Distance $d$ & Terminal velocity $v_{e}$ \\
\hline Desired trajectory & $68.21 \%$ & $7.30 \mathrm{~s}$ & $198.85 \mathrm{~m}$ & $20.09 \mathrm{~m} \cdot \mathrm{s}^{-1}$ \\
LTV-MPC controller & $68.18 \%$ & $7.32 \mathrm{~s}$ & $198.88 \mathrm{~m}$ & $20.10 \mathrm{~m} \cdot \mathrm{s}^{-1}$ \\
PID controller & $64.35 \%$ & $7.68 \mathrm{~s}$ & $208.81 \mathrm{~m}$ & $20.13 \mathrm{~m} \cdot \mathrm{s}^{-1}$ \\
\hline
\end{tabular}


Figure 4 shows the vehicle trajectory of distance and velocity, Figure 5 and Figure 6 shows the vehicle braking force and slip rate, respectively. The regeneration energy, vehicle terminal states are listed in Table 2. In Figure 4 and Table 2, the actual terminal velocity and braking distance error of LTV-MPC controller are $0.01 \mathrm{~m} \cdot \mathrm{s}^{-1}$ and $0.03 \mathrm{~m}$ respectively, and $0.72 \mathrm{~m} \cdot \mathrm{s}^{-1}$ and $9.96 \mathrm{~m}$ of PID controller. The error data shows better tracking performance of the LTV-MPC controller than the PID controller. The distance exceeds the maximum permissible distance $(201.58 \mathrm{~m} \sim 196.73 \mathrm{~m})$ in PID controller, which causes collision possibly. Figure 5 shows the braking force both do not exceed constraints of LTV-MPC and PID controller, however, the PID controller leads to a large tracking error, which is the cause of state tracking error as shown in Figure 4. Figure 6

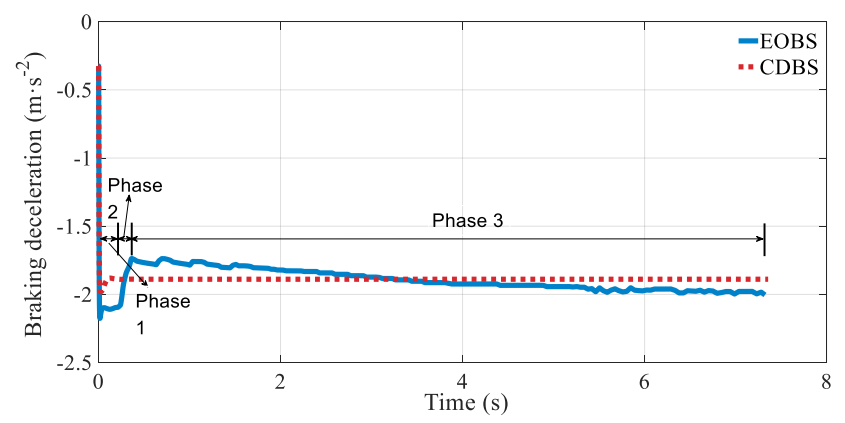

(a) Scenario $\mathrm{A}$ shows the small slip rate of each wheel, which indicates the braking stability is still ensured by employing the LTV-MPC.

As shown in Table 2, the vehicle with the LTV-MPC controller achieves regeneration energy efficiency increase by $3.83 \%$ compared with the PID controller, and decreases by $0.03 \%$ compared with the desired trajectory. Thus, the regeneration energy efficiency of the LTV-MPC controller is superior to the PID controller, because of the excellent tracking performance.

\subsubsection{Regeneration energy efficiency}

The Regeneration energy efficiency of EOBS is evaluated by compared with CDBS in Scenarios A and B. The simulation results are shown in Figure 7 and Table 3.

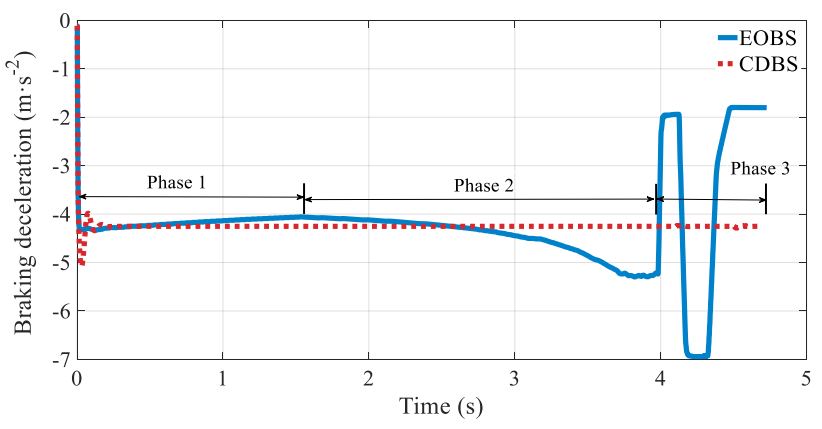

(b) Scenario B

Figure 7 The simulation results of braking deceleration

Table 3 Simulation results of EOBS and CDBS

\begin{tabular}{llcccc}
\hline & & Regeneration energy efficiency $\alpha_{\mathrm{r}}$ & Improvement & Distance $d$ & Terminal velocity $v_{e}$ \\
\hline \multirow{2}{*}{ Scenario A } & EOBS & $68.18 \%$ & $11.42 \%$ & $198.88 \mathrm{~m}$ & $20.10 \mathrm{~m} \cdot \mathrm{s}^{-1}$ \\
& CDBS & $56.76 \%$ & N/A & $198.94 \mathrm{~m}$ & $20.10 \mathrm{~m} \cdot \mathrm{s}^{-1}$ \\
\hline \multirow{2}{*}{ Scenario B } & EOBS & $44.61 \%$ & $3.19 \%$ & $46.97 \mathrm{~m}$ & $0.10 \mathrm{~m} \cdot \mathrm{s}^{-1}$ \\
& CDBS & $41.42 \%$ & N $/ \mathrm{A}$ & $47.13 \mathrm{~m}$ & $0.11 \mathrm{~m} \cdot \mathrm{s}^{-1}$ \\
\hline
\end{tabular}

Figure 7 shows the braking deceleration. The braking deceleration of CDBS is constant (e.g. Scenario A is $1.89 \mathrm{~m} \cdot \mathrm{s}^{-2}$ and Scenario B is $-4.26 \mathrm{~m} \cdot \mathrm{s}^{-2}$, which are the mean deceleration of EOBS ), while EOBS derives a varied deceleration for energy-optimal consideration. Table 3 shows the regeneration energy and vehicle terminal states of EOBS and CDBS. We can observe the vehicle could stay within the defined region in Scenarios A and B, and the proposed EOBS has improved the regeneration energy in Scenario A and Scenario B by $11.42 \%$ and $3.19 \%$ than CDBS.

These above-mentioned results show that the proposed EOBS guarantees the braking safety, while improving regeneration energy, especially in normal braking scenario. Further, the energy-optimal braking mechanism of EVs will be analyzed in detail in Section 4.3.

\subsection{Energy-optimal braking mechanism}

Figures 8-10 are the motor braking force, braking cylinder pressure of HBS (the sum of every wheel), and battery charging power, respectively. The motor efficiency during the regenerative braking and its average value are listed in Table 4.

Scenario A is the normal braking scenario, the mean deceleration is $-1.89 \mathrm{~m} \cdot \mathrm{s}^{-2}$. As shown in Figure 7(a), the deceleration of EOBS including three phases: increasing firstly, then decreasing, and increasing again. The wheel cylinder pressure increases firstly and then drops to approximate zero in the second and third phases (see Figure 9(a)). In the first phase, a large braking deceleration contributes to three advantages: (1) The velocity drops rapidly to decrease the aerodynamic drag (the relationship of aerodynamic drag with velocity is an exponential function); (2) Peak generating power of the motor (see Figure 10(a)) can be fully used for regenerative braking to recover more energy. The second and last 
Energy-Optimal Braking Control Using a Double-Layer Scheme for Trajectory Planning and Tracking of Connected Electric Vehicles

phases of EOBS should be discussed together because these two phases are complementary compared to CDBS. Figures 8(a) and 10(a) illustrate complementary relationship: the motor braking force, battery charging power of EOBS less than CDBS in the second phase, but

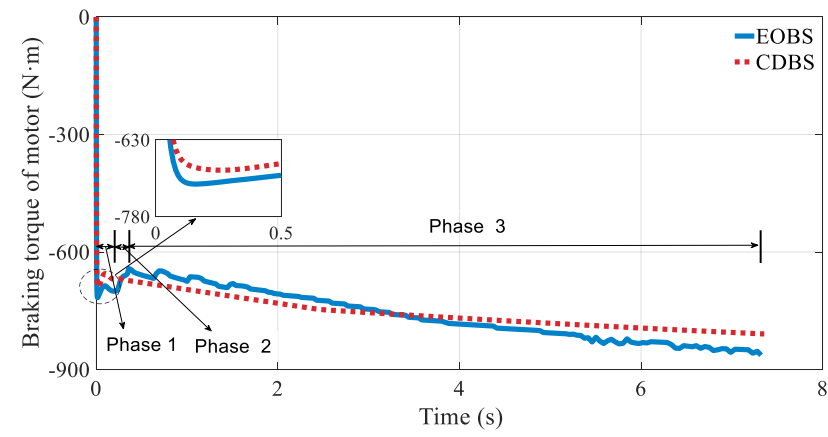

(a) Scenario A

Figure 8 The braking torque of motor

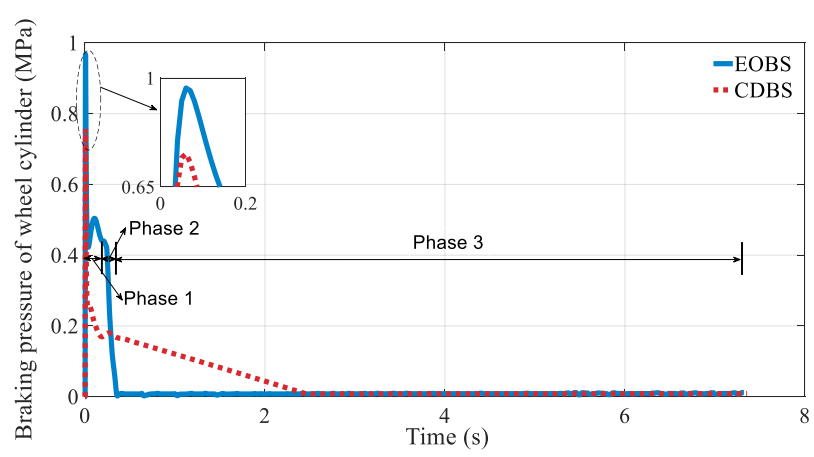

(a) Scenario A

Figure 9 The wheel cylinder pressure of HBS

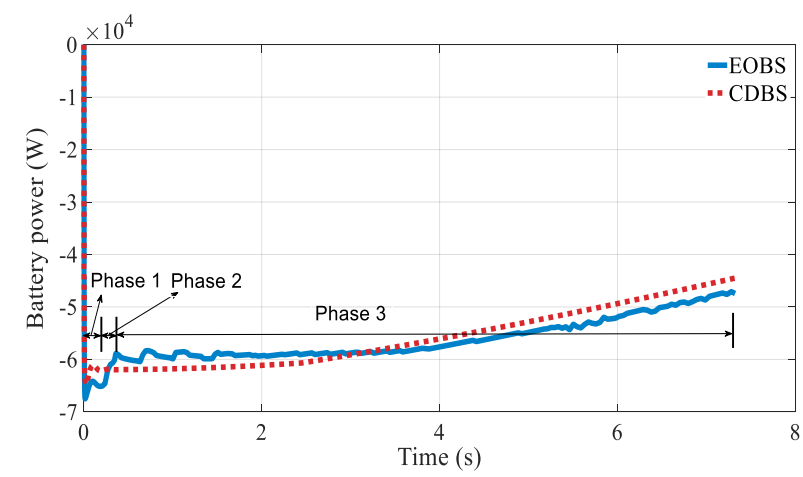

(a) Scenario A larger than CDBS in the last phase. In the second phase, the braking deceleration decreases quickly to reduce friction braking force (see Figure 9(a)). In the last phase, to make full use of motor (see Figures 8(a), 10(a)), the braking deceleration of EOBS increases.

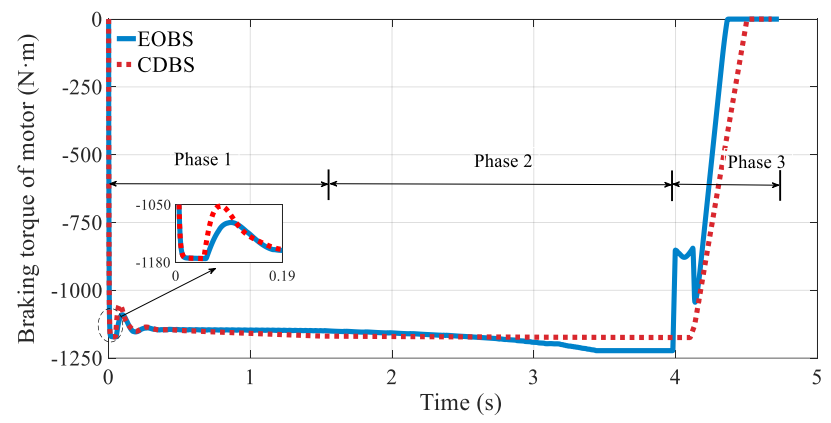

(b)Scenario B

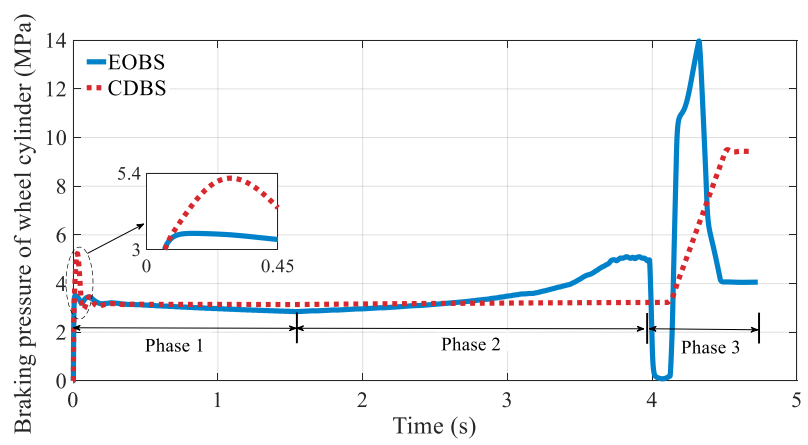

(b) Scenario B

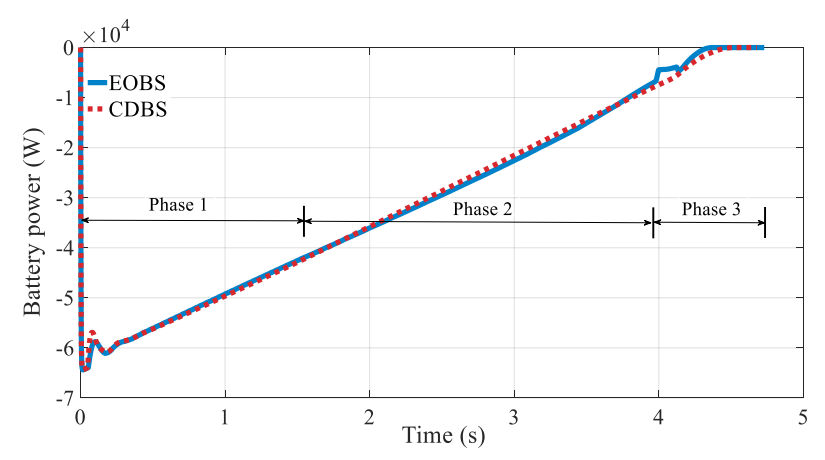

(b) Scenario B

Figure 10 The battery charging power

Table 4 The efficiency of motor

\begin{tabular}{|c|c|c|c|c|}
\hline & & Front axle & Rear axle & \\
\hline & & left/right wheel & left/right wheel & 19. \\
\hline \multirow{2}{*}{ Scenario A } & EOBS & $90.59 \%$ & $87.73 \%$ & $89.16 \%$ \\
\hline & CDBS & $90.55 \%$ & $87.75 \%$ & $89.15 \%$ \\
\hline \multirow{2}{*}{ Scenario B } & EOBS & $77.99 \%$ & $77.93 \%$ & $77.96 \%$ \\
\hline & CDBS & $79.49 \%$ & $79.49 \%$ & $79.49 \%$ \\
\hline
\end{tabular}


Scenario B is the emergency braking scenario, the mean deceleration is $-4.26 \mathrm{~m} \cdot \mathrm{s}^{-2}$. As shown in Figure $7(\mathrm{~b})$, the braking acceleration of EOBS can be divided into three phases same as Scenario A and for the same reasons. The first and second phases are discussed together, in the first phase, the deceleration of EOBS decreases with the velocity drops and increases in the second phase. In the third phase, the braking deceleration decreases because in low-speed operating conditions, the motor generating force is gradually dwindling and replaced by the friction braking force (see Figures 8(b) and 9(b)). As shown in Figure 10(b), the battery charging power of EOBS and CDBS is similar, which causes the regeneration energy improvement of EOBS is not obvious in the emergency braking case (see Table 3).

In addition, Table 4 shows the motor efficiency of EOBS and CDBS. The mean motor efficiency in EOBS is greater than CDBS in Scenario A, but it less than CDBS in Scenario $B$. This phenomenon indicates only focus on the maximum motor efficiency does not mean maximum regeneration energy. The regeneration energy is related to motor generating power and motor efficiency, and the cooptimization of motor generating power and motor efficiency is the key to achieve the energy-optimal braking control. Therefore, the energy-optimal braking control requires reducing vehicle resistance and increasing motor braking force for improving the motor generating power, and operating the motor working at high-efficiency point at the same time.

\section{Conclusions}

This paper presented a double-layer energy-optimal braking strategy to improve the regeneration energy using shared braking intention. First, the energy-optimal braking trajectory is derived by distance-based dynamic programming in the upper-layer. Second, the linear timevarying model predictive controller is formulated to follow the desired trajectory which derived from the upper-layer, to ensures regeneration energy efficiency, braking safety, and comfort. The simulation results demonstrate the proposed energy-optimal braking strategy achieves prominent regeneration energy improvement than regular constant deceleration braking strategy, and the trajectory tracking performance of the linear time-varying model predictive controller is superior to regular PID controller. The analysis of the energy-optimal braking mechanism indicates the optimal regeneration energy operation is required to reduce the vehicle resistance and friction braking force while ensures motor working at high-efficiency point as much as possible.

\section{Declaration}

\section{Acknowledgements}

We gratefully acknowledges the support of Efficient Mobility Control Lab, Southeast University.

\section{Funding}

Supported by National Nature Science Foundation of China (Grants No. U1664258, 51805081 and 51975118), National Key R\&D Program in China (Grants No. 2016YFB0100906 and 2016YFD0700905).

\section{Availability of data and materials}

The datasets supporting the conclusions of this article are included within the article.

\section{Authors' contributions}

YG provided fundamental ideas and all support conditions of this research. HD was in charge of the trial and wrote the manuscript. WZ assisted with building up the framework of the research. LX and YW assisted the trial and simulations. FW and YL conducted proof reading and made some critical revisions. All authors read and approved the final manuscript.

\section{Competing interests}

The authors declare no competing financial interests.

\section{Consent for publication}

Written informed consent for publication was obtained from all participants.

\section{Ethics approval and consent to participate}

Not applicable

\section{References}

[1] G B Ning, X Lu, L J Zhang, et al. Method of electric powertrain matching for battery-powered electric cars. Chinese Journal of Mechanical Engineering, 2013, 26(3): 483-491.

[2] Z P Cano, D Banham, S Ye, et al. Batteries and fuel cells for emerging electric vehicle markets. Nature Energy, 2018, 3(4): 279-289.

[3] A Adepetu, S Keshav. The relative importance of price and driving range on electric vehicle adoption: Los Angeles case study. Transportation, 2017, 44(2): 353-373.

[4] L li, Y B Zhang, C Yang, et al. Model predictive control-based efficient energy recovery control strategy for regenerative braking system of hybrid electric bus. Energy conversion and management, 2016, 111: 299-314.

[5] Y M Gao Y, L P Chen, M Ehsani. Investigation of the effectiveness of regenerative braking for EV and HEV. SAE Technical Paper 1999, No. 1999-01-2910.

[6] J Hellgren, E Jonasson. Maximisation of brake energy regeneration in a hybrid electric parallel car. International Journal of Electric and Hybrid Vehicles, 2007,1(1): 95-121. 
[7] J Joy, S Ushakumari. Regenerative braking mode operation of a threephase H-bridge inverter fed PMBLDC motor generator drive in an electric bike. Electric Power Components and Systems, 2018;46(19): 2174-2188.

[8] K Kiddee, W Keyoonwong, W Khan-Ngern. An HSC/battery energy storage system-based regenerative braking system control mechanism for battery electric vehicles. IEEJ Transactions on Electrical and Electronic Engineering, 2019, 14(3): 457-466.

[9] J Z Zhang, C Lv, J Gou, et al. Cooperative control of regenerative braking and hydraulic braking of an electrified passenger car. Proceedings of the Institution of Mechanical Engineers, Part D: Journal of Automobile Engineering, 2012, 226(10): 1289-1302.

[10] J G Guo. Development of regenerative braking for electric vehicles in China: a review. International Journal of Electric and Hybrid Vehicles, 2015, 7(2): 120-138

[11] F C Sun, W Liu, H W He, et al. An integrated control strategy for the composite braking system of an electric vehicle with independently driven axles. Vehicle System Dynamics, 2016, 54(8): 1031-1052.

[12] W Xu, H Chen, J M Wang, et al. Velocity optimization for braking energy management of in-wheel motor electric vehicles. IEEE Access, 2019, 7: 66410-66422.

[13] W Li W, H Du, W Li. Driver intention based coordinate control of regenerative and plugging braking for electric vehicles with in-wheel PMSMs. IET Intelligent Transport Systems, 2018, 12(10): 1300-1311.

[14] Y Lian, Y Zhao, L Hu, et al. Longitudinal collision avoidance control of electric vehicles based on a new safety distance model and constrained-regenerative-braking-strength-continuity braking force distribution strategy. IEEE Transactions on Vehicular Technology, 2015, 65(6): 4079-4094.

[15] L H Björnsson, S Karlsson. The potential for brake energy regeneration under Swedish conditions. Applied Energy, 2016, 168: 75-84.

[16] G Zhao, X Peng. Variable structure control strategy research on regenerative braking for a brushless DC motor driven electric bus cruising downhill. Journal of Advanced Manufacturing Systems, 2014 13(4): 223-236.

[17] G Chen, M Hua, C Zong, et.al. Comprehensive chassis control strategy of FWIC-EV based on sliding mode control. IET Intelligent Transport Systems, 2018, 13(4): 703-713.

[18] B Liu, L Li, X Wang, et.al. Hybrid electric vehicle downshifting strategy based on stochastic dynamic programming during regenerative braking process. IEEE Transactions on Vehicular Technology, 2018, 67(6): 4716-4727.

[19] A Vahidi, A Sciarretta. Energy saving potentials of connected and automated vehicles. Transportation Research Part C: Emerging Technologies, 2018, 95: 822-843.

[20] Y C Lin, HLT Nguyen. Adaptive Neuro-Fuzzy Predictor-Based Control for Cooperative Adaptive Cruise Control System. IEEE Transactions on Intelligent Transportation Systems, 2020, 21(3):1054-1063

[21] SE Li, Q Guo, S Xu, et.al. Performance enhanced predictive control for adaptive cruise control system considering road elevation information. IEEE Transactions on Intelligent Vehicles. 2017, 2(3): 150-160.

[22] Ding F, Jin H. On the optimal speed profile for eco-driving on curved roads. IEEE Transactions on Intelligent Transportation Systems, 2018 19(12): 4000-4010.

[23] He X, Liu H X, Liu X. Optimal vehicle speed trajectory on a signalized arterial with consideration of queue. Transportation Research Part C: Emerging Technologies 2015, 61: 106-120.
[24] V H Johnson. Battery performance models in ADVISOR. Journal of Power Sources, 2002;110(2): 321-329.

[25] C S N Kumar, S C Subramanian. Cooperative control of regenerative braking and friction braking for a hybrid electric vehicle. Proceedings of the Institution of Mechanical Engineers, Part D: Journal of Automobile Engineering, 2016, 230(1): 103-116.

[26] W C Zhuang, X Zhang, D Li, et.al. Mode shift map design and integrated energy management control of a multi-mode hybrid electric vehicle. Applied Energy, 2017, 204: 476-488.

[27] P Falcone, F Borrelli, H E Tseng, et.al. Linear time-varying model predictive control and its application to active steering systems: Stability analysis and experimental validation. International Journal of Robust and Nonlinear Control: IFAC-Affiliated Journal, 2008, 18(8): 862-875.

[28] F Kuhne F, W FLages, J Silva. Model predictive control of a mobile robot using linearization. Proceedings of mechatronics and robotic, 2004:525-530.

[29] Z Chen, C C Mi, R Xiong, et.al. Energy management of a power-split plug-in hybrid electric vehicle based on genetic algorithm and quadratic programming. Journal of Power Sources, 2014, 248: 416426.

[30] D Chakraborty D, A K Nandi . Finding optimal deceleration with serial regenerative braking of electric vehicle using a multi-objective genetic algorithm. IEEE 1st International Conference on Power Electronics, Intelligent Control and Energy Systems (ICPEICES), IEEE, 2016: 1-6.

\section{Biographical notes}

Hao-xuan Dong, born in 1993, is currently a PhD candidate at the School of Mechanical Engineering, Southeast University, China. He received his M.S. degree on vehicle engineering from Chang 'an University, China, in 2018. His research interests include connected vehicles, vehicle energy management, eco-driving control.

Tel: +86-18363623668; E-mail: donghaox@foxmail.com

Wei-chao Zhuang, born in 1990, is currently an Assistant Professor at the School of Mechanical Engineering, Southeast University, China. He received the B.S. and Ph.D. degrees on mechanical engineering from Nanjing University of Science and Technology, China, in 2012 and 2017, respectively. His current research interests include vehicle dynamics and control, optimal control, clean energy vehicles, connected vehicles, and multi-agent control.

Tel: +86-15951860337; E-mail: wezhuang@seu.edu.cn

Guo-dong Yin, born in 1976, is currently a Professor at the School of Mechanical Engineering, Southeast University, China. He received the Ph.D. degree on vehicle engineering from Southeast University, China, in 2007. His current research interests include vehicle dynamics and control, connected vehicles, and multiagent control.

Tel: +86-13913879060; E-mail: ygd@seu.edu.cn

Li-wei Xu, born in 1986, is currently a Research Associate at the School of Mechanical Engineering, Southeast University, China. $\mathrm{He}$ received the $\mathrm{Ph} . \mathrm{D}$. degree on mechanical engineering from Southeast University, China, in 2019. His current research interests include vehicle dynamics and control, autonomous vehicles, and connected vehicles. 
Tel: +86-18602548629; E-mail: eagil123@hotmail.com

Yan Wang, born in 1992, is currently a PhD candidate at the School of Mechanical Engineering, Southeast University, China. $\mathrm{He}$ received the M.S. degree on vehicle engineering from Nanjing University of Aeronautics and Astronautics, in 2018. His current research interests include vehicle state and parameter estimation, automotive active safety control.

Tel: +86-13645165076; E-mail: yanwangiv@outlook.com

Fa-an Wang, born in 1990, is currently a $\mathrm{PhD}$ candidate at the School of Mechanical Engineering, Southeast University, China. $\mathrm{He}$ received the M.S. degree on agricultural engineering from Kunming University of Science and Technology, China, in 2017. His current research interests include vehicle cooperative localization and control, intelligent connected vehicles.

Tel: +86-18317710956; E-mail: faanwang@seu.edu.cn

Yan-bo Lu, born in 1995, is currently a $\mathrm{PhD}$ candidate at the School of Mechanical Engineering, Southeast University, China. He received his B.S. degree on mechanical engineering from Nanjing University of Aeronautics and Astronautics, China, in 2017. His research interests include vehicle dynamics and control, vehicle rollover prevention.

Tel: +86-13451826598; E-mail: 230198035@seu.edu.cn 


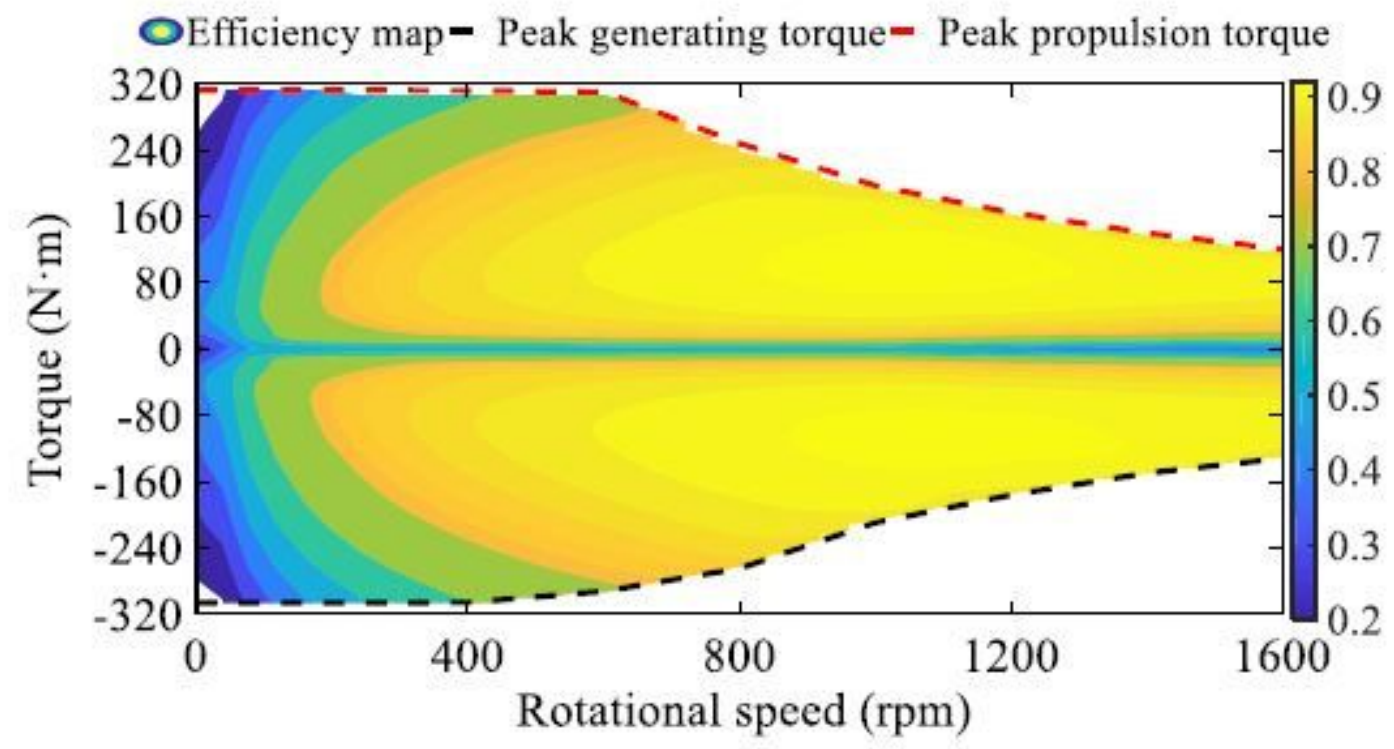

\section{Figure 1}

The efficiency map and peak torque of IWM

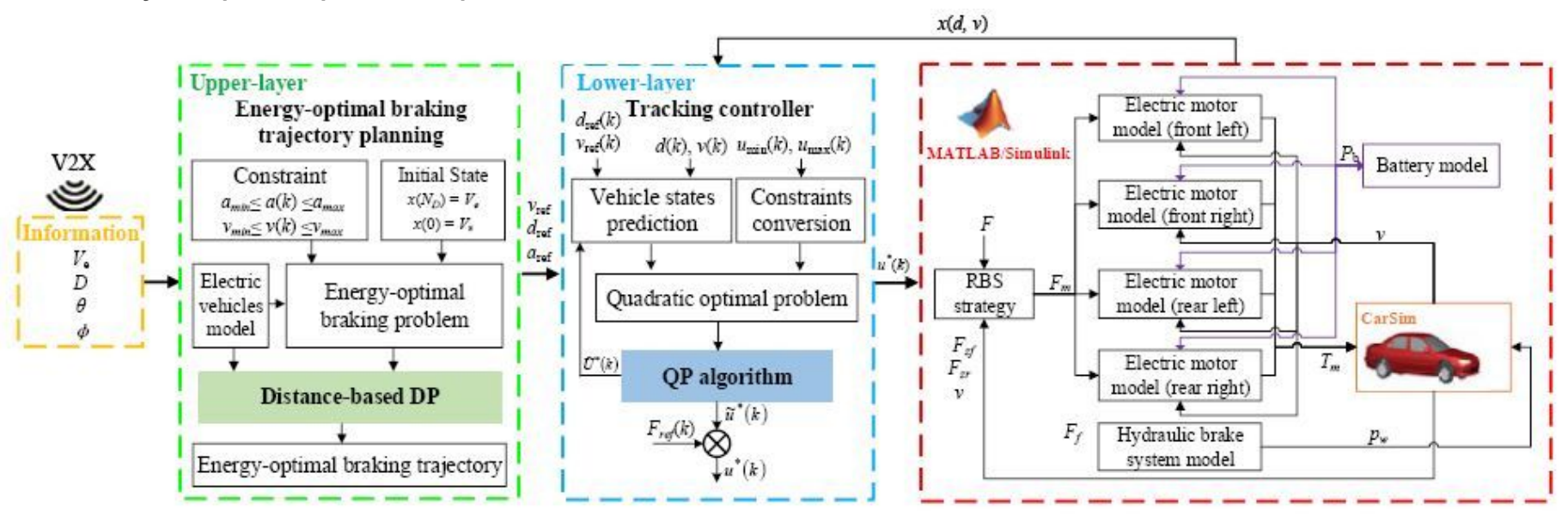

\section{Figure 2}

The EOBS scheme 


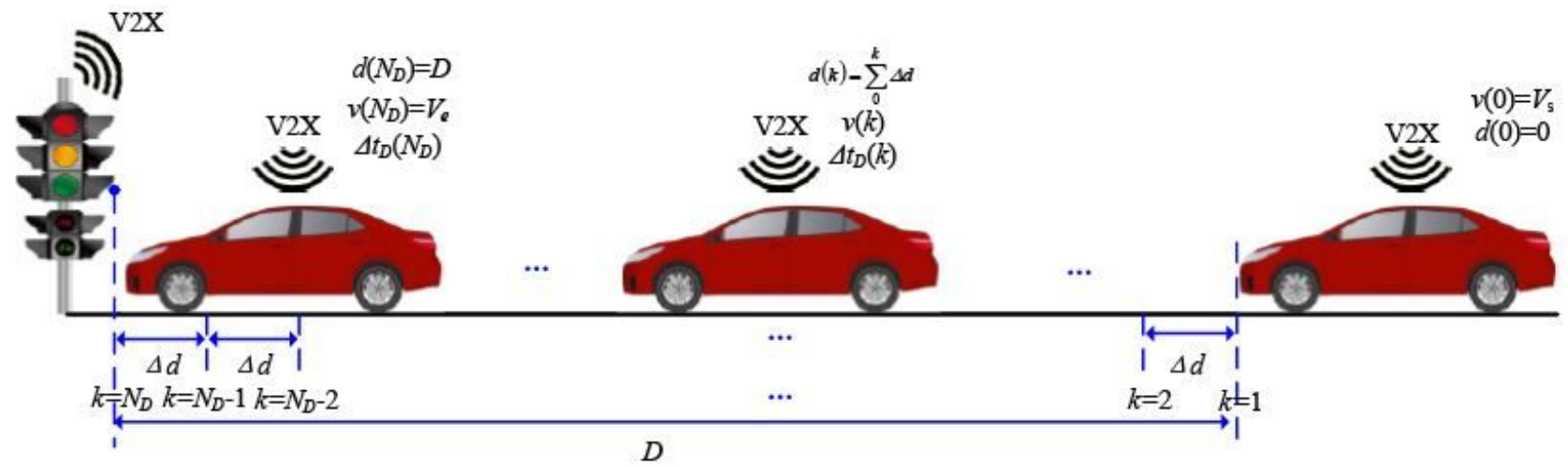

\section{Figure 3}

The process of braking

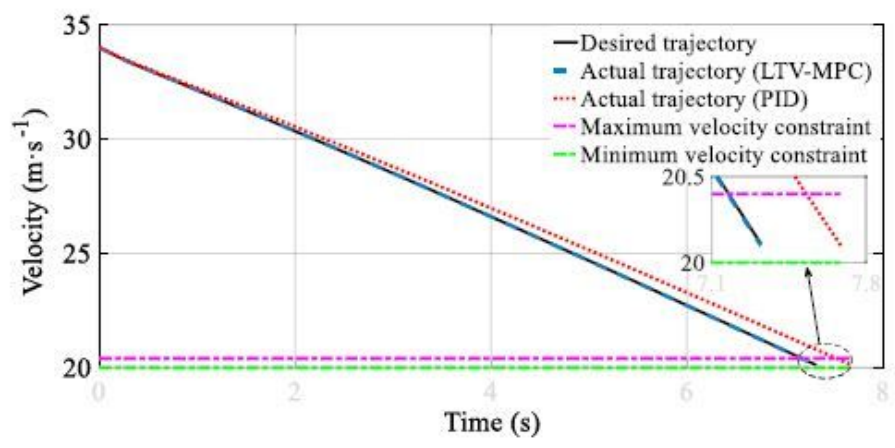

(a) Velocity trajectory

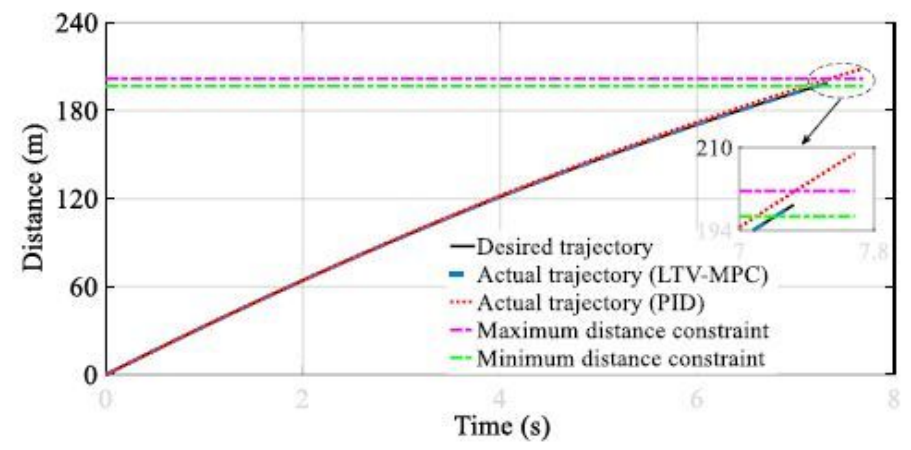

(b) Distance trajectory

\section{Figure 4}

The braking trajectory of EVs

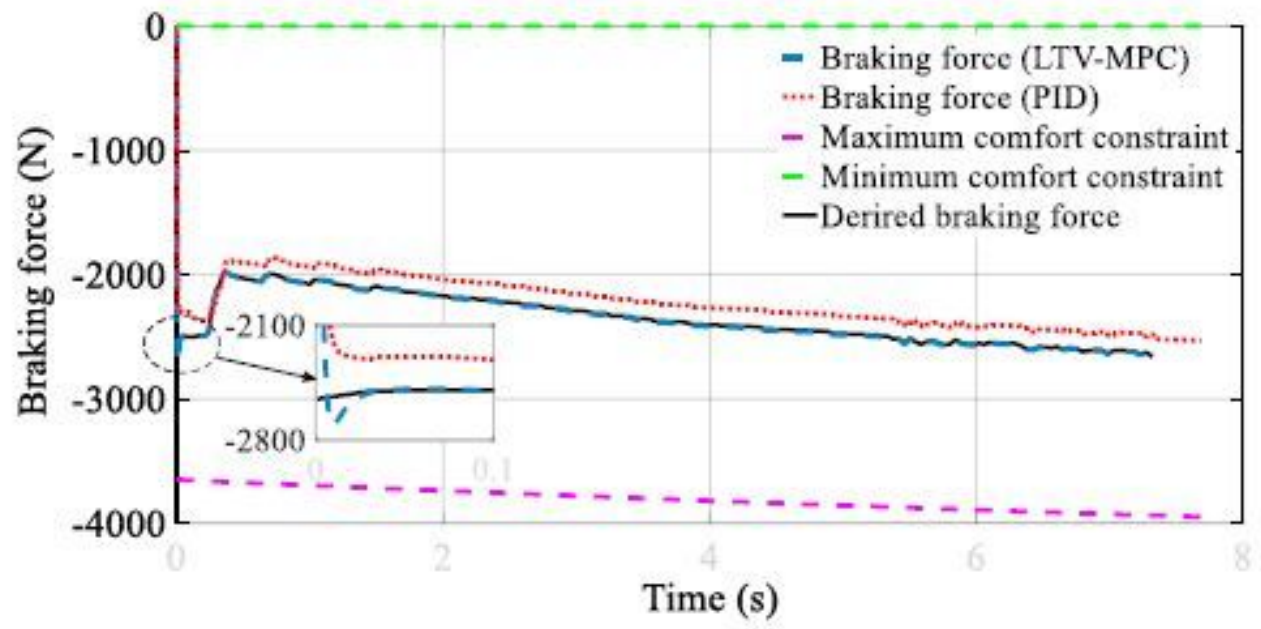

\section{Figure 5}




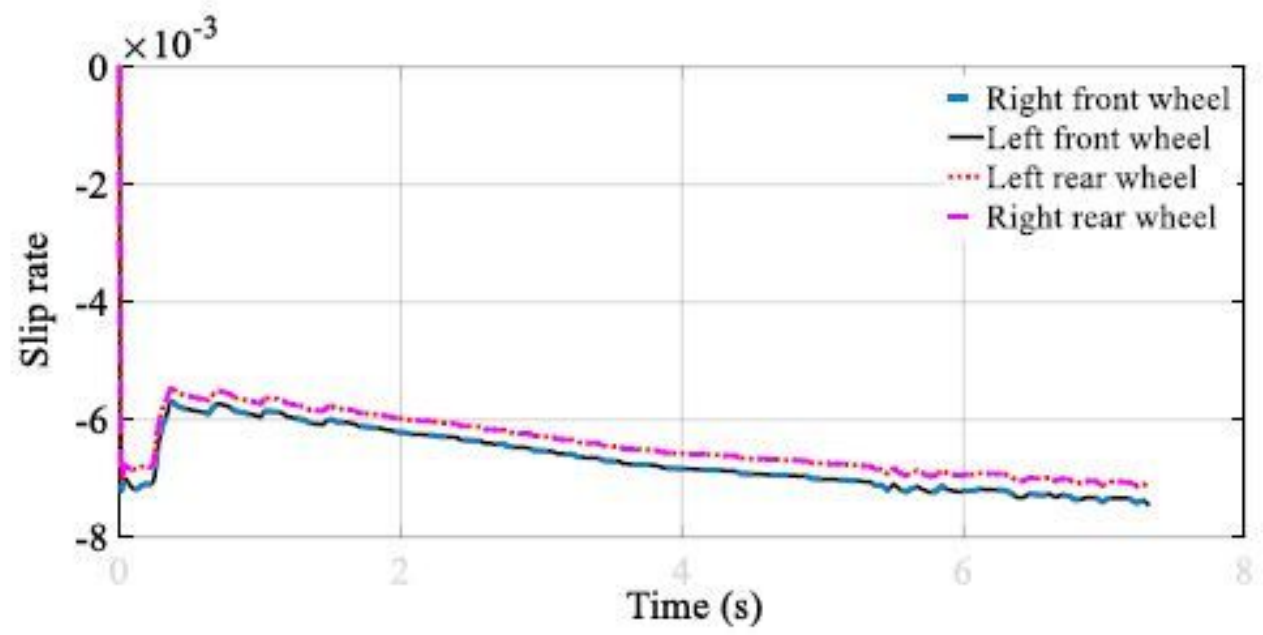

\section{Figure 6}

The slip rate of EVs by LTV-MPC

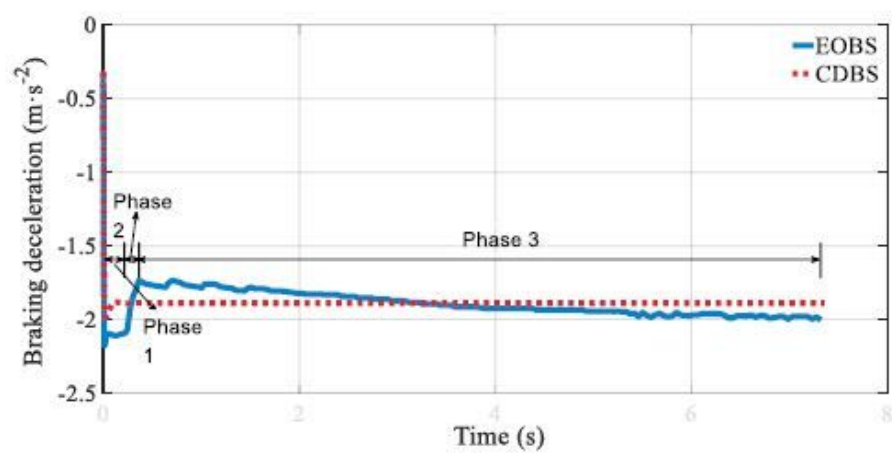

(a) Scenario $\mathrm{A}$

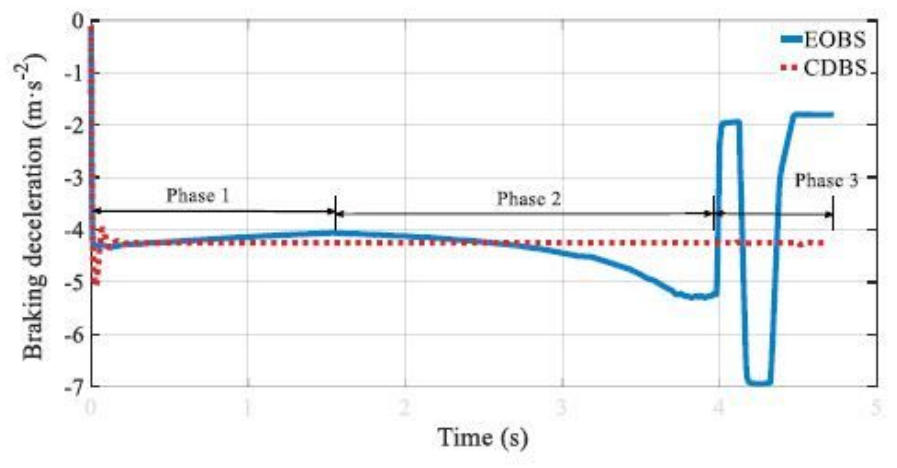

(b) Scenario B

\section{Figure 7}

The simulation results of braking deceleration

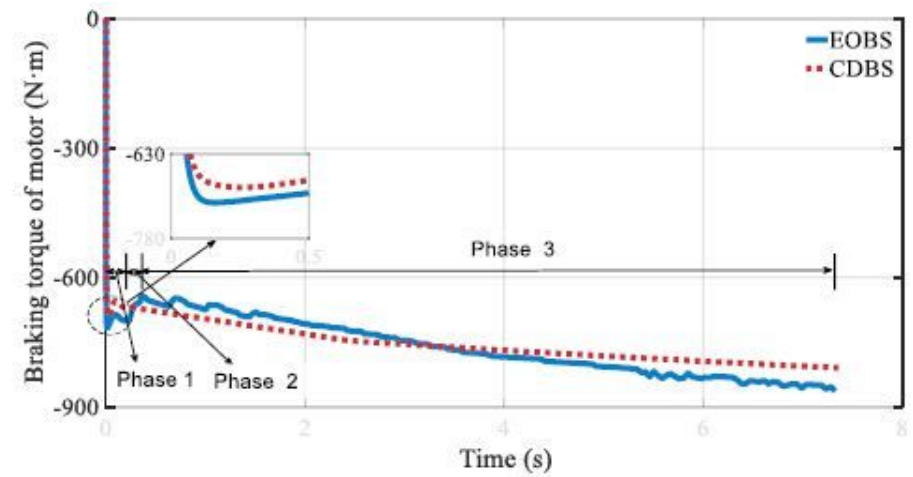

(a) Scenario A

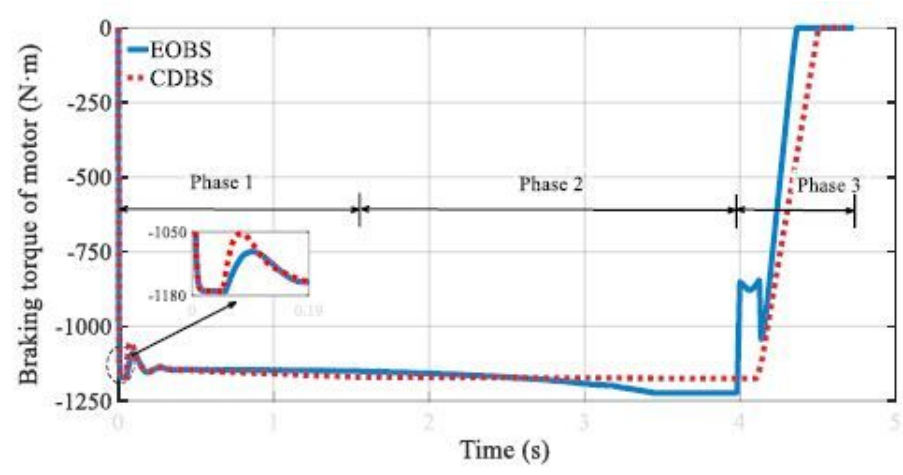

(b)Scenario B

Figure 8

The braking torque of motor 


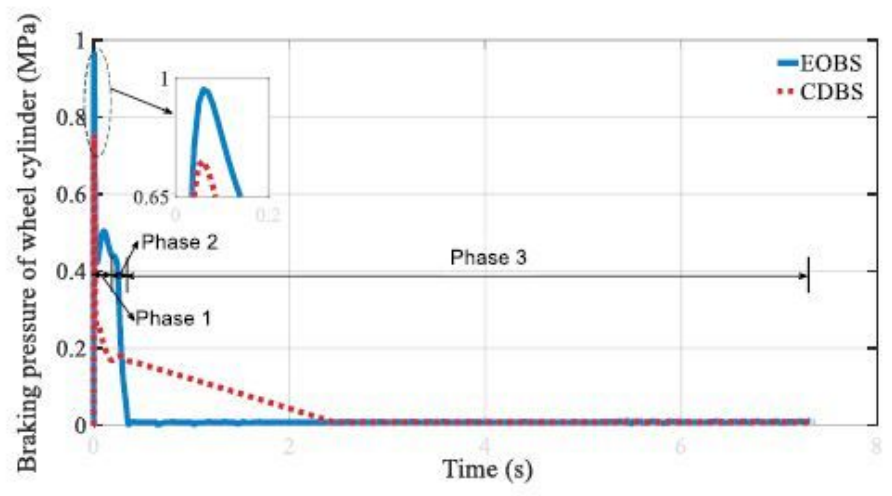

(a) Scenario A

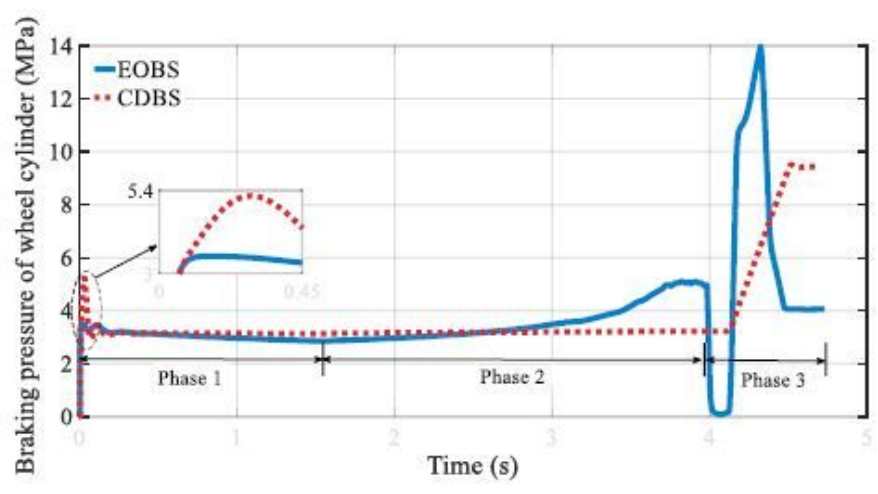

(b) Scenario B

\section{Figure 9}

The wheel cylinder pressure of HBS

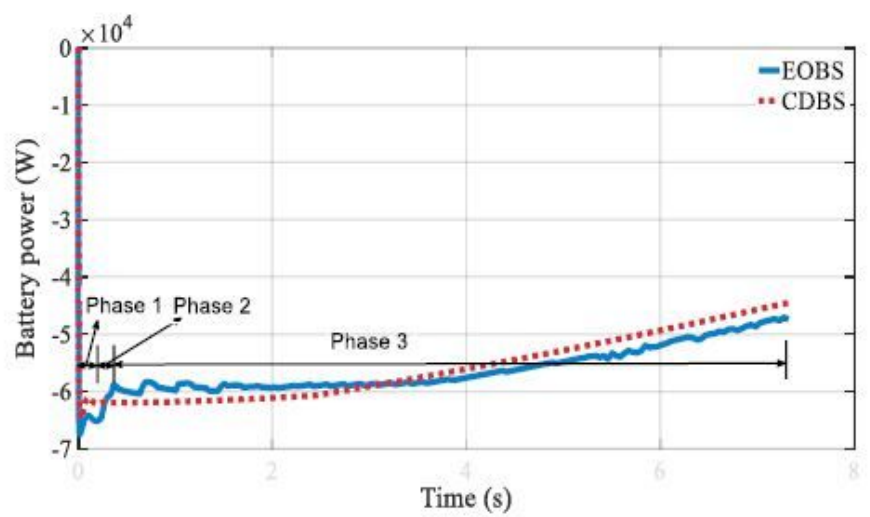

(a) Scenario $\mathrm{A}$

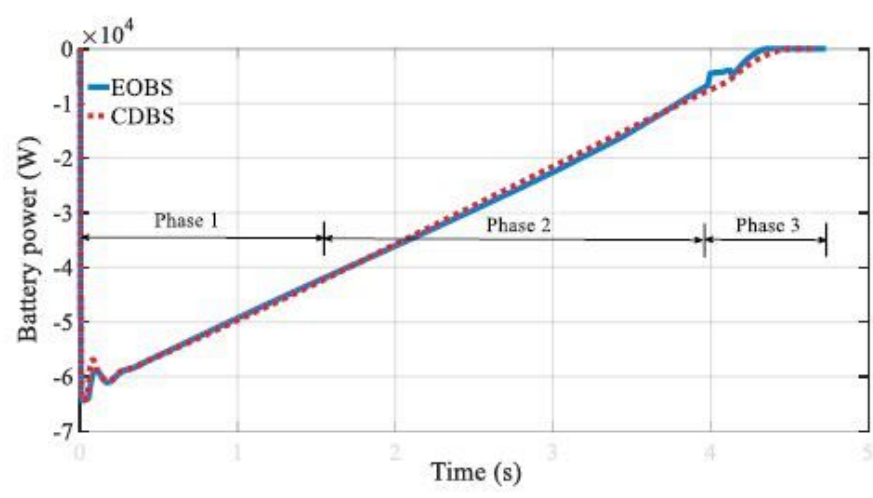

(b) Scenario B

\section{Figure 10}

The battery charging power 